\title{
Mucocele: Clinical Characteristics and Outcomes in 46 Operated Patients
}

\author{
Diogo Barreto Plantier ${ }^{1}$ Deusdedit Brandão Neto ${ }^{1}$ Fabio de Rezende Pinna ${ }^{1}$ Richard Louis Voegels ${ }^{1}$ \\ ${ }^{1}$ Department of Otolaryngology, Hospital das Clínicas da Faculdade \\ de Medicina da Universidade de São Paulo, Sao Paulo, Brazil \\ Int Arch Otorhinolaryngol 2019;23:88-91.

\begin{abstract}
Address for correspondence Diogo Barreto Plantier, Fellow, Departamento de Otorrinolaringologia, Hospital das Clínicas da Faculdade de Medicina da Universidade de São Paulo, Av. Dr. Enéas de Carvalho Aguiar, 255 - $6^{\circ}$ andar - sala 6167, Sao Paulo, SP 05403-000, Brazil (e-mail: diogoplantier@gmail.com).
\end{abstract}

Abstract
Keywords
- mucocele
- paranasal sinus
diseases
- chronic rhinosinusitis
- endoscopic sinus
surgery
- marsupialization

Introduction Paranasal sinus mucocele is a benign, expansive lesion associated with paranasal sinus obstruction. It affects mostly adults, and is most common in the frontal and ethmoidal sinuses.

Objective To evaluate outcomes in patients undergoing surgical treatment for paranasal sinus mucocele.

Methods Retrospective review of medical records of patients treated for paranasal sinus mucocele at the ENT department of a tertiary care hospital between 2005 and 2016.

Results Forty-six patients underwent surgical treatment of paranasal sinus mucocele. The mean age was 50.1 years, and $56.5 \%$ were male. The most prevalent symptom was pain, and the frontal sinus was most commonly affected. The vast majority of patients (89.1\%) underwent endoscopic sinus marsupialization; $10.9 \%$ required combined open and endoscopic access. Seven recurrences occurred.

Conclusion Sinus mucocele is an expansive disease that primarily affects the frontal sinus of adult patients. In most cases, endoscopic surgery is an effective treatment modality.

\section{Introduction}

Paranasal sinus mucocele is a benign disease characterized by mucus filling of a paranasal sinus, with possible expansion. ${ }^{1}$ Lesions are usually lined by pseudostratified columnar epithelium, but, in some cases, may be associated with areas of squamous epithelium, inflammatory cell infiltration, bone resorption, and new bone formation. ${ }^{2}$ It is most common in adults, and usually originates from a sinus obstruction, which may be spontaneous or secondary to factors such as trauma or past paranasal sinus surgery. ${ }^{3}$ Sinus mucocele is rare in the pediatric population, and such cases should prompt investigation of cystic fibrosis. ${ }^{4}$

The most common locations are the frontal and ethmoidal sinus (frontoethmoidal distribution). Comparatively, the maxillary and sphenoid sinus are less often affected. ${ }^{5}$ Other rare locations have been described in the literature, including the crista galli, ${ }^{6}$ Onodi cell, ${ }^{7}$ pneumatized middle turbinate (concha bullosa), ${ }^{8}$ Haller cell, ${ }^{9}$ and even in the pneumatized uncinate process. ${ }^{10}$

Clinically, besides the usual symptoms of sinusitis, patients with mucocele usually present specifically with headache or facial pain. ${ }^{3}$ If intracranial or orbital extension has occurred, patients may report visual disturbances or exhibit proptosis. ${ }^{11}$

Surgery is the only curative treatment, and the endoscopic marsupialization technique can solve most cases. ${ }^{12}$ This procedure is usually done under general anesthesia. In patients without anxiety, septated mucoceles, or neo-osteogenesis, in-office drainage with only topical anesthesia has been reported. ${ }^{13}$ Most surgically treated patients in whom the periosteum is preserved have good outcomes, including bone regeneration. ${ }^{14}$ received

January 24, 2018

accepted

May 26, 2018

published online

October 24, 2018
DOI https://doi.org/

10.1055/s-0038-1668126. ISSN $1809-9777$.
Copyright (e) 2019 by Thieme Revinter

Publicações Ltda, Rio de Janeiro, Brazil
License terms

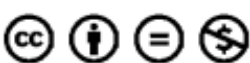


The present study describes the clinical characteristics of a series of patients who underwent surgical treatment for paranasal sinus mucocele at the ear, nose, and throat (ENT) service of a tertiary care hospital and provides an update on the natural history and outcomes of this disease.

\section{Methodology}

Data on patients who underwent surgical treatment for paranasal sinus mucocele at the study facility between January 2005 and December 2016 were collected retrospectively. The parameters of interest were age, sex, possible etiology, and presentation (sinonasal symptoms, facial deformity, visual changes, proptosis, headache, and facial pain).

All patients underwent computed tomography (CT) to determine the location of mucocele and presence of orbital or intracranial extension, as well as for surgical planning. In cases of cranial or orbital extension, magnetic resonance imaging (MRI) was performed for additional evaluation.

Treatment generally consisted of wide opening of the affected paranasal sinus with marsupialization of the mucocele while preserving its lining mucosa. Procedures were performed exclusively by endonasal sinus surgery (ESS) technique or via a combination of open surgical access and the endonasal route.

The intraoperative findings and any complications were recorded. Postoperative follow-up lasted a minimum of 5 years. Patients were seen weekly until evidence of successful healing was identified; depending on the progression of healing, follow-up became monthly, semiannual, and annual thereafter.

The study was approved by the local Research Ethics Committee.

\section{Results}

From January 2005 to December 2016, 46 patients with paranasal sinus mucocele (26 men, 20 women; mean age 50.1 years, range $13-84$ years) underwent surgery at the service where the study was conducted. Pain was the most common symptom, reported by 28 patients, followed by facial deformity $(n=18)$, proptosis $(n=14)$, and visual disturbances $(n=6)$.

On imaging (CT or MRI), 20 patients exhibited dehiscence or extension of the mucocele to the orbital space. In addition, 12 patients had skull base extension or erosion, one had anterior maxillary wall erosion, one had posterior maxillary wall erosion, and two had maxillary floor extension (-Fig. 1).

The most commonly affected locations, in descending order, were the frontal sinus (43.5\%), maxillary sinus (19.6\%), frontoethmoidal sinus $(8.7 \%)$, ethmoidal sinus (6.5\%), and sphenoidal sinus (4.3\%). Multiple-sinus involvement was detected in six patients: two with large mucoceles extending to the ipsilateral maxillary, ethmoidal, and frontal sinuses and four with concomitant maxillary and ethmoidal sinus involvement. Surprisingly, in one case, we identified a frontoethmoidal sinus mucocele on the right side and a large concha bullosa mucocele on the left (-Fig. 2). Another patient presented with an isolated concha bullosa mucocele.

Of the 46 patients, 10 (21.7\%) had a history of chronic rhinosinusitis. History of cranial trauma was reported by eight patients (17.4\%), and nine (19.6\%) had undergone previous paranasal sinus surgery. One patient had been treated for leukemia, one had an IgG4-associated pseudotumor of the lacrimal duct, and another had undergone radiation therapy for nasal lymphoepithelioma.

All patients underwent surgery under general anesthesia. Forty-one $(89.1 \%)$ had endoscopic sinus marsupialization alone, while five (10.9\%) required a combination of open access and ESS. All patients who required open access were treated for frontal mucocele (three for the first time and two for recurrence).

Intraoperative complications occurred in three cases: one minor lamina papyracea injury and two cases of heavy bleeding controlled during surgery, with no need to interrupt the procedure and no need for transfusion.

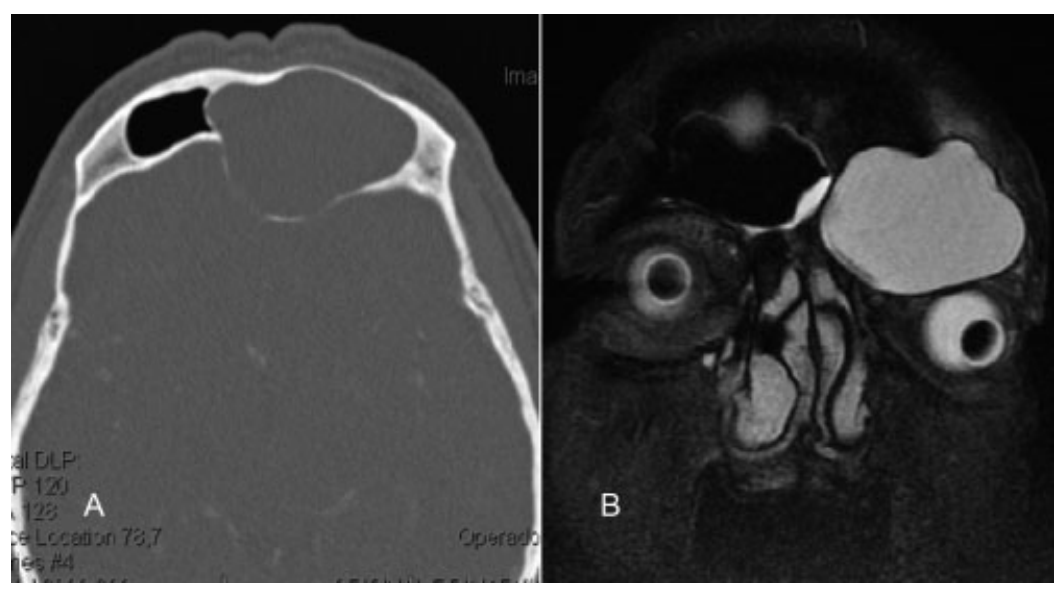

Fig. 1 (A) Axial CT image of the paranasal sinus showing an extensive, homogeneous mucocele in the left frontal sinus, with apparent bone defect of the posterior wall. (B) MRI showing extensive T2 hyperintensities indicative of mucocele in the left frontal sinus, with associated extension to the ipsilateral orbit. 


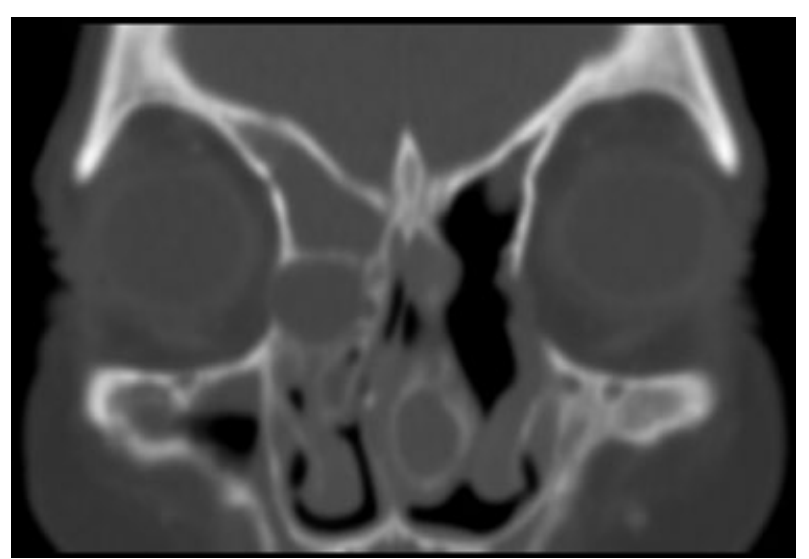

Fig. 2 Coronal CT image of the paranasal sinuses with bone window. A large frontoethmoidal mucocele is visible on the right side, and a concha bullosa mucocele on the left.

At the time of writing, seven patients (15.2\%) had developed recurrences, five with frontal and two with frontoethmoidal mucocele.

\section{Discussion}

In this paper, we reviewed a series of 46 patients who underwent surgical treatment of paranasal sinus mucocele at a university-affiliated tertiary care center between January 2005 and December 2016.

According to the literature, patients with paranasal sinus mucocele usually present between the fifth and sixth decades of life. ${ }^{3,11,13-17}$ Our patients had a median age of 50.1 years, which is consistent with this profile.

Analysis of the most prevalent symptoms in our sample revealed that $60.9 \%$ of patients reported pain before surgery, which is also consistent with current knowledge. ${ }^{13,17}$ Ocular symptoms, such as visual disturbance and proptosis, are due to the close relationship of all paranasal sinuses with the orbit. ${ }^{11}$ Accordingly, other prevalent symptoms were proptosis, present in 14 patients (30.4\%), and visual disturbance, present in six (13\%). Of those with proptosis, 11 had a frontal sinus mucocele, two had an ethmoidal mucocele, and one had a maxillary sinus mucocele. Orbital or intracranial extension are most often associated with frontal mucocele. ${ }^{17}$

All patients underwent CT scan and, in case of cranial or orbital invasion, MRI. Imaging showed involvement of the orbit in 20 patients (43.5\%), of the skull base in 12 (26.1\%), and of the anterior maxillary wall, posterior wall and sinus floor in one patient each. Extension of mucocele to the orbit and skull base is very prevalent in the literature, with the orbit being more commonly affected. ${ }^{5,13,14,17}$ According to current data, erosion of the orbit and skull can occur in up to $83.3 \%$ and $55.5 \%$ of cases respectively. ${ }^{5}$

In our sample, the frontal sinus was the most commonly affected site, as in previous studies. ${ }^{13,14,17}$ of 27 patients with frontal mucocele, 20 had isolated frontal lesions, five had lesions that extended to the ethmoid, and two involved the ethmoid and maxillary sinuses (one of these patients also had concha bullosa mucocele). Unlike in previous studies, however, the maxillary sinus was more commonly affected than the ethmoid. In the sample as a whole, 15 patients had some extension to the maxillary sinus, while 14 had involvement of the ethmoid.

When mucocele has a possibly identifiable etiological cause, it is known as secondary mucocele. ${ }^{17}$ Among these causes, a history of craniofacial trauma or paranasal sinus surgery is frequently reported. ${ }^{3}$ Overall, $19.6 \%$ of our patients had a history of surgery, while $17.4 \%$ reported previous craniofacial trauma.

The only effective treatment for paranasal sinus mucocele is surgery. ${ }^{14}$ Intraoperatively, purulent discharge, which characterizes so-called mucopyocele, was identified in $80.4 \%$ of patients. Of these, $37.8 \%$ had received antibiotics before surgery, which reinforces the notion that antibiotics are ineffective as a single treatment option for mucocele.

Endoscopic marsupialization could be performed successfully in almost all cases (89.1\%). Image-guided navigation is not available at the service where the study was performed. Therefore, in cases of frontal mucocele in which marsupialization could not be performed fully endoscopically, we used combined open and endoscopic access. This was required especially for lateral frontal mucoceles and cases with extensive inferior neo-osteogenesis in the frontal recess or septations in the mucocele. All patients who required a combined approach had frontal sinus involvement.

In a recent meta-analysis of frontal sinus mucocele treatment, 542 patients who underwent surgery between 2002 and 2014 were assessed. Of these, 53.9\% were treated endoscopically, $37.5 \%$ via external access, and $8.8 \%$ via a combined approach. ${ }^{18}$ In our study, 27 patients required surgical approach to the frontal sinus; of these, only $18.5 \%$ required combined access. Mucoceles present in the other paranasal sinuses were effectively treated by ESS. In short, ESS proved to be effective in the majority of mucoceles, even those affecting the frontal sinus.

Regarding intraoperative complications, two patients had heavy bleeding, which did not require interruption of the surgical procedure or blood transfusion. Another patient had injury of the lamina papyracea without periorbital injury or exposure of orbital contents. These three complications occurred in endoscopically treated patients and represent $6.5 \%$ of these surgeries, a complication rate above that described in the literature. ${ }^{18}$ After surgery, all patients reported symptom relief.

On average, recurrence of paranasal sinus mucocele develops in the fourth year after surgery. ${ }^{3,17}$ At the time of writing, recurrence had been detected in seven patients (15.2\%), which is within the range described in the literature (9\% to $23.5 \%)^{3,17}$ These seven were operated by endoscopy, one of whom required combined access. Based on location, despite wide opening, thorough postoperative care, and healing of the frontal recess, five recurrences developed in the frontal sinus and two were frontoethmoidal. This rate of recurrence may be associated with the fact that we prioritized ESS over combined access whenever possible, as well as with the absence of image-guided navigation. Longer postoperative follow-up is needed to evaluate all patients 
in the series for potential recurrence, particularly those operated more recently.

\section{Conclusions}

This paper reported a series of patients with paranasal sinus mucocele and reviewed the diagnosis and treatment of this unusual entity. A presenting complaint of progressive, localized facial pain, especially in patients between the fifth and sixth decades of life and when associated with facial deformities or visual disturbances, should raise suspicion of a possible diagnosis of mucocele. Beyond physical examination and nasal endoscopy, CT scan and, in select cases, MRI are essential to confirm diagnosis of mucocele, determine the location of the lesion, and evaluate possible involvement of the orbit or skull base.

Endoscopic marsupialization has proven to be an effective therapeutic modality, and its use should be encouraged. Some patients with frontal mucocele will require combined access, especially when the lesion is located more laterally or associated with abundant neo-osteogenesis, which can hinder opening of the frontal recess.

\section{References}

1 Canalis RF, Zajtchuk JT, Jenkins HA. Ethmoidal mucoceles. Arch Otolaryngol 1978;104(05):286-291

2 Lund VJ, Milroy CM. Fronto-ethmoidal mucocoeles: a histopathological analysis. J Laryngol Otol 1991;105(11):921-923

3 Devars du Mayne M, Moya-Plana A, Malinvaud D, Laccourreye O, Bonfils P. Sinus mucocele: natural history and long-term recurrence rate. Eur Ann Otorhinolaryngol Head Neck Dis 2012;129 (03):125-130

4 Nicollas R, Facon F, Sudre-Levillain I, Forman C, Roman S, Triglia JM. Pediatric paranasal sinus mucoceles: etiologic factors, management and outcome. Int J Pediatr Otorhinolaryngol 2006;70(05):905-908
5 Har-El G. Endoscopic management of 108 sinus mucoceles. Laryngoscope 2001;111(12):2131-2134

6 Cervantes SS, Lal D. Crista galli mucocele: endoscopic marsupialization via frontoethmoid approach. Int Forum Allergy Rhinol 2014;4(07):598-602

7 Victores A, Foroozan R, Takashima M. Recurrent Onodi cell mucocele: rare cause of 2 different ophthalmic complications. Otolaryngol Head Neck Surg 2012;146(02):338-339

8 Al-Sebeih KH, Bu-Abbas MH. Concha bullosa mucocele and mucopyocele: a series of 4 cases. Ear Nose Throat J 2014;93(01): 28-31

9 Lee JH. Large Haller cell mucocele leading to maxillary sinusitis. Ear Nose Throat J 2015;94(4-5):E53-E54

10 Burrows SA. Mucocele of a pneumatised uncinate process: first reported case. J Laryngol Otol 2011;125(06):635-637

11 Zainine R, Loukil I, Dhaouadi A, et al. Complications ophtalmiques des mucocèles rhino-sinusiennes. J Fr Ophtalmol 2014;37(02): 93-98

12 Kennedy DW, Josephson JS, Zinreich SJ, Mattox DE, Goldsmith MM. Endoscopic sinus surgery for mucoceles: a viable alternative. Laryngoscope 1989;99(09):885-895

13 Barrow EM, DelGaudio JM. In-office drainage of sinus Mucoceles: An alternative to operating-room drainage. Laryngoscope 2015; 125(05):1043-1047

14 Terranova P, Karligkiotis A, Digilio E, et al. Bone regeneration after sinonasal mucocele marsupialization: What really happens over time? Laryngoscope 2015;125(07):1568-1572

15 Nazar R, Naser A, Pardo J, Fulla J, Rodríguez-Jorge J, Delano PH. Manejo endoscópico de mucoceles de senos paranasales: experiencia en 46 pacientes. Acta Otorrinolaringol Esp 2011;62(05): 363-366

16 Busaba NY, Salman SD. Maxillary sinus mucoceles: clinical presentation and long-term results of endoscopic surgical treatment. Laryngoscope 1999;109(09):1446-1449

17 Scangas GA, Gudis DA, Kennedy DW. The natural history and clinical characteristics of paranasal sinus mucoceles: a clinical review. Int Forum Allergy Rhinol 2013;3(09):712-717

18 Courson AM, Stankiewicz JA, Lal D. Contemporary management of frontal sinus mucoceles: a meta-analysis. Laryngoscope 2014;124 (02):378-386 